\title{
A Preliminary Analysis of Interdependence in Multiagent Systems
}

\author{
Ronal Singh, Tim Miller, Liz Sonenberg \\ University of Melbourne, Melbourne, Australia. \\ ronals@student.unimelb.edu.au, tmiller@unimelb.edu.au, \\ 1.sonenberg@unimelb.edu.au
}

\begin{abstract}
Designers of human-agent systems use the term "interdependence," drawing on the work of organisational theorists and sociologists that is set in a human context. In this paper, we extend the agent systems analysis by semi-formally defining several types of task and agent interdependence that are introduced in the organisation theory literature. We illustrate how knowledge of different types of interdependence can assist designers in choosing appropriate coordination mechanisms between agents.
\end{abstract}

Keywords: interdependence, interdependence types, task interdependence, agent interdependence

\section{Introduction}

To tackle the intricacies of designing agents capable of exhibiting collaboration with humans, Johnson et al. [4] propose the Coactive Design Method. This method allows designers to identify, analyse, implement and evaluate interdependence relationships between agents involved in joint activities. Interdependence has been highlighted as the central organising principle of Coactive Design Method, and has been defined as "the set of complementary relationships that two or more parties rely on to manage...dependencies in joint activity" [4]. This definition of interdependence builds on insights from earlier studies of human systems $[10,11,12]$ that interdependence is more complex than simply mutual dependence: it is about relationships and includes consideration of the purpose of those relationships being to manage dependencies in joint activity. Interdependence relationships must be complementary, and help identify what common ground [6] should comprise.

While the Coactive Design Method explicitly considers interdependence as an organising principle, two additional fruitful contributions can be made in this area: (1) the extension of the concept of interdependence beyond tasks; and (2) a more rigorous analysis of interdependence. According to sociologists and organisational theorists $[7,8,10,11,12]$ numerous types of interdependence exist, including interdependence between agents, tasks, and goals. This paper takes inspiration from these works. Similarly, we go further than the formalisms offered 
in $[1,9]$ for bilateral dependence. In this paper, we semi-formally define the concepts of task and agent interdependence. The more formal treatment provides a more fine-grained analysis of different types of interdependence that can be used to assist designers in developing computational models of interdependence between humans and agents involved in joint activity. In the context of multi agent systems, coordination is the management of interaction between agents [5], and is also linked to the notion of agent autonomy [3]. One of the results of the studies performed by organisational theorists is that different types of interdependencies require different coordination mechanisms $[12,8]$. Following the rigorous analysis of interdependence in this paper, we conjecture that a sound knowledge of the type of interdependency involved in various multi-agent scenarios will assist designers in the choice of appropriate coordination mechanisms.

The structure of this paper is as follows. Sections 2 and 3 discuss the different types of interdependence. In Section 4, we apply the formal definitions to a scenario discussed by Johnson et al. [4]. Section 5 compares our work with two closely related papers $[1,4]$, and Section 6 concludes the paper.

\section{Task Interdependence}

In this section, we present our formal definition of task interdependence.

We assume that agents are systems capable of autonomous actions on the environment. Tasks, which may require one or more actions, are performed to achieve goals. Goals represent the state of the environment that the agent wants to achieve. Task interdependence exists when the value generated from performing each task is different when the tasks are performed together versus when the tasks are performed separately [7].

Definition 1 (Task). The following grammar defines tasks:

$$
t::=\text { act }\left|t_{1} ; t_{2}\right| t_{1} \| t_{2}\left|t_{1}\right| \mid t_{2}
$$

in which act is an atomic action of an agent, $t_{1} ; t_{2}$ represents sequential execution such that $t_{1}$ executes before $t_{2}, t_{1} \| t_{2}$ represents interleaved execution, and $t_{1} \| t_{2}$ represents true concurrent execution. The difference between interleaving and true concurrency is that in interleaving, actions cannot execute simultaneously, whereas in true concurrency, actions (and entire tasks) can execute simultaneously. We use the shorthand $t_{1} \odot t_{2}$ to mean composition of $t_{1}$ and $t_{2}$ using any of the operators ;, $\|$, or $\| \mid$, and the shorthand $\overline{t_{1} \odot t_{2}}$ to mean the complement of (any composition other than) $t_{1} \odot t_{2}$; for example, $\overline{t_{1} ; t_{2}}$ means either $t_{1}\left\|t_{2}, t_{1} \mid\right\| t_{2}$, or $t_{2} ; t_{1}$. The operators $\|$ and $\| \mid$ are commutative, so the complement $\overline{t_{1} \| t_{2}}$ does not contain $t_{2} \| t_{1}$, and similarly for $\|$. We consider the semantics of interleaved concurrency to be that of CSP [2]. For true concurrency, we consider the semantics to be that of independent actions [13].

Definition 2 (Task Value). The tangible value generated by executing a task $(t)$ in some context $(c)$ is measured by $\mathcal{V}_{c}(t)$. 
Definition 3 (Task Independence). Tasks are independent if and only if the value generated by executing the composed tasks is the same as the sum of the value of each task in isolation. That is, we gain nothing by composing the two tasks. More formally:

$$
\text { TaskIndep }\left(t_{1}, t_{2}\right) \Leftrightarrow \mathcal{V}_{c}\left(t_{1}\right)+\mathcal{V}_{c}\left(t_{2}\right)=\mathcal{V}_{c}\left(t_{1} \odot t_{2}\right)
$$

We can generalise this to $n \geq 2$ tasks:

$$
\begin{aligned}
& \text { TaskIndep }\left(t_{1}, \ldots, t_{n}\right) \Leftrightarrow \mathcal{V}_{c}\left(t_{1}\right)+\mathcal{V}_{c}\left(t_{2}\right)=\mathcal{V}_{c}\left(t_{1} \odot t_{2}\right) \wedge \\
& \mathcal{V}_{c}\left(t_{1}\right)+\mathcal{V}_{c}\left(t_{3}\right)=\mathcal{V}_{c}\left(t_{1} \odot t_{3}\right) \wedge \ldots \wedge \mathcal{V}_{c}\left(t_{n-1}\right)+\mathcal{V}_{c}\left(t_{n}\right)=\mathcal{V}_{c}\left(t_{n-1} \odot t_{n}\right)
\end{aligned}
$$

which is equivalent to

$$
\text { TaskIndep }\left(t_{1}, t_{2}\right) \wedge \text { TaskIndep }\left(t_{1}, t_{3}\right) \wedge \ldots \wedge \operatorname{TaskIndep~}\left(t_{n-1}, t_{n}\right)
$$

Example 1 (Obstacle detection in human agent team (Independence)). One task required in the DARPA Robotics Challenge is obstacle detection [4]. Consider two tasks, SenseObsHum and SenseObsRob. The human detects obstacles via a user interface while the robot uses sensors. The two tasks are independent because the value of identifying an obstacle together is the same as both the human and robot identifying them independently. Given that the context $c$ is detecting obstacles, independence is explained by applying Equation 1:

$\mathcal{V}_{c}($ SenseObsHum $)+\mathcal{V}_{c}($ SenseObsRob $)=\mathcal{V}_{c}($ SenseObsHum $\odot$ SenseObsRob $)$

Definition 4 (Task Dependence). Composed execution of dependent tasks (using any composition operator) generates a value greater than independent execution of those tasks. If task $t_{2}$ is dependent on $t_{1}$ then formally:

$$
\text { TaskDep }\left(t_{1} \mid t_{2}\right) \Leftrightarrow \mathcal{V}_{c}\left(t_{1} \odot t_{2}\right)>\mathcal{V}_{c}\left(t_{1}\right)+\mathcal{V}_{c}\left(t_{2}\right)
$$

As with task independence, task dependence can be generalised to $n \geq 2$ tasks.

Definition 5 (Soft/Hard Task dependence). A soft (hard) task dependence is when a task is dependent on another task, but the value of executing the dependent task is non-zero (zero). Formally:

$$
\begin{aligned}
\operatorname{SoftTaskDep}\left(t_{1} \mid t_{2}\right) & \Leftrightarrow \operatorname{TaskDep}\left(t_{1} \mid t_{2}\right) \wedge \mathcal{V}_{c}\left(t_{2}\right)>0 \\
\operatorname{HardTaskDep}\left(t_{1} \mid t_{2}\right) & \Leftrightarrow \operatorname{TaskDep}\left(t_{1} \mid t_{2}\right) \wedge \mathcal{V}_{c}\left(t_{2}\right)=0
\end{aligned}
$$

Definition 6 (Task Interdependence). Two tasks are interdependent if there is a two-way dependency between the tasks. Formally:

$$
\text { TaskInterdep }\left(t_{1}, t_{2}\right) \Leftrightarrow \text { TaskDep }\left(t_{1} \mid t_{2}\right) \wedge \text { TaskDep }\left(t_{2} \mid t_{1}\right)
$$

This can be generalised to $n \geq 2$ cases as is done for task independence.

Definition 6 captures the essence of interdependence but not the differences between the different types of interdependencies. Therefore, next we formalise several types of interdependencies, based on organisational theory [8].

\subsection{Types of Task Interdependence}

According to organisational theorists and sociologists $[7,8,11]$, there are four types of task interdependence: sequential, reciprocal, team, and pooled. In the 
following sections, we formalise these definitions. We give definitions for pairs of tasks, but each is generalisable to $n \geq 2$, as done for independence.

Definition 7 (Reciprocal Task Interdependence). The value of reciprocally interdependent tasks is strictly greater than the value generated by any other composition. The notion of temporal lag between tasks is captured by the interleaved execution of the tasks. Formally:

$$
\text { TaskInterdep }\left(t_{1}, t_{2}\right) \wedge \mathcal{V}_{c}\left(t_{1} \| t_{2}\right)>\mathcal{V}_{c}\left(\overline{t_{1} \| t_{2}}\right)
$$

Example 2 (Writing a paper (Reciprocal Interdependence)). Consider one person writing a paper and another proof reading it and providing feedback, represented by the tasks WritePaper and ReadPaper. While these tasks can be executed sequentially, in many cases, more value is generated if the reader provides feedback on individual sections through the writing. But doing these truly concurrently would require the reader to read over the writer's shoulder, which is presumably not as valuable. Applying Equation 6:

$$
\mathcal{V}_{c}(\text { WritePaper } \| \text { ReadPaper })>\mathcal{V}_{c}(\overline{\text { WritePaper } \| \text { ReadPaper }})
$$

Definition 8 (Team Task Interdependence). Team task interdependence exists for joint activities - that is, when agents must jointly execute one or more actions. There is no temporal lag between task executions, because tasks are executed simultaneously. For such tasks, the value of a truly concurrent composition is strictly greater than any other composition. Formally:

$$
\text { TaskInterdep }\left(t_{1}, t_{2}\right) \wedge \mathcal{V}_{c}\left(t_{1} \| \mid t_{2}\right)>\mathcal{V}_{c}\left(\overline{t_{1}|| t_{2}}\right)
$$

Example 3 (Cooperative object transportation (Team Interdependence)).

Two robots are to move a table that is too heavy for one robot to move by itself. Consider two tasks MoveEnd1 and MoveEnd2, where MoveEnd1 means that one robot will lift and move the table at one of the two ends. Only true concurrent execution of the tasks will be able to move the table. Applying Equation 7:

$$
\mathcal{V}_{c}(\text { MoveEnd1 }|| \mid \text { MoveEnd2 })>\mathcal{V}_{c}(\overline{\text { MoveEnd } 1|| \mid \text { MoveEnd2 }})
$$

Definition 9 (Sequential Task Interdependence). The value generated by sequential composition of two interdependent tasks is greater than any other composition. Formally:

$$
\text { TaskInterdep }\left(t_{1}, t_{2}\right) \wedge \mathcal{V}_{c}\left(t_{1} ; t_{2}\right)>\mathcal{V}_{c}\left(\overline{t_{1} ; t_{2}}\right)
$$

Note that this is not simply task dependence, because the value of performing the first task can be higher if the second task is performed; e.g. picking up a hose is only valuable if the hose is subsequently attached.

Definition 10 (Pooled Task Interdependence). Composition of tasks exhibiting pooled interdependence result in greater value than of executing the tasks independently. This is simply equivalent to the initial definition of interdependence, but with no constraint on how the tasks are composed. Each task contributes its share towards the group outcome, and failure of any task means that the goal will not be achieved. This is a weak form of interdependence. 


\section{Agent Interdependence}

In the previous section, task interdependence is agnostic on who executes the task or who gains the value of the execution. In this section, we consider how agents can be independent, dependent, and interdependent. Throughout this section, we use the same grammar to define tasks, and $a_{i} . t_{j}$ to mean that agent $a_{i}$ executes task $t_{j}$. The semantics remains the same, essentially ignoring the agent prefixes, which are just labels. This means that for composed tasks, if two agents $a_{1}$ and $a_{2}$ both execute the same action act, this is a synchronised event (they must execute together). We treat such synchronisations as joint actions.

According to Puranam et al. [7], agent interdependence results when "...the reward to $A$ from $A$ 's actions depends on the actions taken by $B$...". We formalise the agent's reward using the notion of utility. We introduce soft and hard agent dependence and interdependence. Hard dependence means that the agent cannot complete its tasks independently (utility is zero), while for soft dependence, it can complete the task, but receives more utility if it works with other agents.

Definition 11 (Agent Utility). An agent's utility when executing a task $t$ in a context $c$ is defined as $\mathcal{U}_{c}^{a_{i}}(t)$. Each agent has their own utility function, which could be associated with measures such as how quickly the agent finishes a task.

Definition 12 (Agent Independence). Two agents, $a_{1}$ and $a_{2}$, are independent if each agent's utility is not affected by the actions of the other agent. Formally:

$$
\begin{aligned}
& \text { AgentIndep }\left(a_{1}, a_{2}\right) \Leftrightarrow \forall t_{1}, t_{2}: \mathcal{U}_{c}^{a_{1}}\left(a_{1} . t_{1} \odot a_{2} . t_{2}\right)=\mathcal{U}_{c}^{a_{1}}\left(a_{1} . t_{1}\right) \wedge \\
& \mathcal{U}_{c}^{a_{2}}\left(a_{1} . t_{1} \odot a_{2} . t_{2}\right)=\mathcal{U}_{c}^{a_{2}}\left(a_{2} . t_{2}\right)
\end{aligned}
$$

Definition 13 (Agent Dependence). Agent $a_{2}$ is dependent on $a_{1}$ for task $t_{2}$ if the utility of $a_{2}$ increases when agent $a_{1}$ executes a task $t_{1}$. Dependence is related to specific tasks; that is, it is possible to be dependent on another agent for some tasks, but not others. Formally:

$$
\operatorname{AgentDep}\left(a_{1} \mid a_{2}, t_{1}, t_{2}\right) \Leftrightarrow \mathcal{U}_{c}^{a_{2}}\left(a_{1} . t_{1} \odot a_{2} . t_{2}\right)>\mathcal{U}_{c}^{a_{2}}\left(a_{2} . t_{2}\right)
$$

Agent dependence can also be defined for some unnamed task as:

$$
\operatorname{Agent} \operatorname{Dep}\left(a_{1} \mid a_{2}\right) \Leftrightarrow \exists t_{1}, t_{2}: \operatorname{Agent} \operatorname{Dep}\left(a_{1} \mid a_{2}, t_{1}, t_{2}\right)
$$

Definition 14 (Soft/Hard Agent Dependence). Agent $a_{2}$ has a soft (hard) dependence on agent $a_{1}$ if the utility of $a_{2}$ at its task is non-zero (zero) and the utility of $a_{2}$ increases when agent $a_{1}$ executes one of its tasks. Formally:

$$
\begin{aligned}
& \text { SoftAgentDep }\left(a_{1} \mid a_{2}, t_{1}, t_{2}\right) \Leftrightarrow \\
& \qquad \text { AgentDep }\left(a_{1} \mid a_{2}\right) \wedge 0<\mathcal{U}_{c}^{a_{2}}\left(a_{2} \cdot\left(t_{1} \odot t_{2}\right)\right)<\mathcal{U}_{c}^{a_{2}}\left(a_{1} \cdot t_{1} \odot a_{2} \cdot t_{2}\right) \\
& \text { HardAgentDep }\left(a_{1} \mid a_{2}, t_{1}, t_{2}\right) \Leftrightarrow \operatorname{AgentDep}\left(a_{1} \mid a_{2}\right) \wedge \mathcal{U}_{c}^{a_{2}}\left(a_{2} \cdot\left(t_{1} \odot t_{2}\right)\right)=0
\end{aligned}
$$

Definition 15 (Agent Interdependence). Agents $a_{2}$ and $a_{1}$ are interdependent if there is a two-way dependence between the agents. More formally:

$$
\begin{aligned}
& \text { AgentInterdep }\left(a_{1}, a_{2}, t_{1}, t_{2}, t_{3}, t_{4}\right) \Leftrightarrow \\
& \qquad \operatorname{Agent} \operatorname{Dep}\left(a_{1} \mid a_{2}, t_{1}, t_{2}\right) \wedge \operatorname{Agent} \operatorname{Dep}\left(a_{2} \mid a_{1}, t_{3}, t_{4}\right)
\end{aligned}
$$


This definition demonstrates that agent interdependence is not merely task interdependence over utilities, because two pairs of dependent tasks between the agents is enough to establish interdependence. This is consistent with Puranum et al.'s view [7] that task interdependence is neither necessary nor sufficient to establish agent interdependence. As with agent dependence, we can omit the tasks $t_{1}$ to $t_{4}$ to avoid explicitly naming the tasks that are (inter)dependent.

Definition 16 (Soft/Hard Agent Interdependence). Soft (hard) agent interdependence between two agents exists when both agents have soft (hard) dependence on each other. Formally:

$$
\begin{aligned}
\text { SoftAgentInterdep }\left(a_{1}, a_{2}\right) & \Leftrightarrow \\
\text { SoftAgentDep }\left(a_{1} \mid a_{2}\right) & \wedge \operatorname{SoftAgentDep}\left(a_{2} \mid a_{1}\right) \\
\text { HardAgentInterdep }\left(a_{1}, a_{2}\right) & \Leftrightarrow \\
\text { HardAgentDep }\left(a_{1} \mid a_{2}\right) & \wedge \operatorname{HardAgentDep}\left(a_{2} \mid a_{1}\right)
\end{aligned}
$$

Example 4 (Object transportation (Hard Agent Interdependence)). In Example 3, two robots $\left(r_{1}\right.$ and $\left.r_{2}\right)$ moved a heavy table. Assume that the utility is: $\mathcal{U}_{c}^{a_{i}}(t)=$ total_time and the context $c$ is object transportation. The utilities of the individual tasks are zero because the robots can not move the table alone. When the two robots cooperatively move the table, the utility of each increases:

$$
\begin{gathered}
\mathcal{U}_{c}^{r_{1}}\left(r_{1} . \text { MoveEnd } 1\right)=\mathcal{U}_{c}^{r_{2}}\left(r_{2} . \text { MoveEnd } 2\right)=0 \\
\mathcal{U}_{c}^{r_{1}}\left(r_{1} . \text { MoveEnd } 1 \| r_{2} . \text { MoveEnd } 2\right)>\mathcal{U}_{c}^{r_{1}}\left(r_{1} .(\text { MoveEnd } 1 \odot \text { MoveEnd } 2)\right) \wedge \\
\mathcal{U}_{c}^{r_{2}}\left(r_{1} . \text { MoveEnd } 1 \| r_{2} . \text { MoveEnd } 2\right)>\mathcal{U}_{c}^{r_{2}}\left(r_{2} .(\text { MoveEnd } 1 \odot \text { MoveEnd } 2)\right)
\end{gathered}
$$

\section{Example}

One of the tasks in the DARPA Robotics Challenge was for the simulated robot to grasp a hose [4]. The robot and the operator each lacked the capability to correctly position the robot's hand for the robot to grasp the hose. Consider two tasks PosHandOp and PosHandRb, which represents the operator and the robot positioning the robot's hand respectively. The two tasks are interdependent because we need to execute both tasks to be able to grasp the hose. This follows that the agents are interdependent on each other.

The tasks could execute sequentially: the robot executes its task before the operator, and the tasks repeat until the robot's hand is positioned correctly. Alternatively, the tasks can be reciprocally executed such that while the robot tries to position its hand, the operator intervenes as necessary.

For a truly sequential execution of the tasks, the designer must program the robot to only receive operator command after the robot has positioned the hand. However, to enable reciprocal or interleaved execution, the designer needs to provision the robot to accept and execute commands from the operator as it is engaged in its task, and provide the necessary interfaces for the operator. The choice of the execution method depends on the objective of the designer. 
For example, if the objective is to minimise the temporal lag between the tasks, then reciprocal execution is appropriate. That is:

$$
\mathcal{V}_{c}(\text { PosHandOp } \| \text { PosHandRb })>\mathcal{V}_{c}(\text { PosHandOp; PosHandRb })
$$

As such, the robot should be designed to accept operator commands that override its own positioning tasks as it tries to position its hand.

The formalism provides designers with a more systematic and rigorous way to analyse the possibilities, and to weigh up different design decisions. Further, depending on the type of task interdependence, the designer may need to implement different coordination mechanisms between the agents.

\section{Discussion}

In this section, we discuss how our definitions align with two closely related papers: Castlefranchi et al.'s [1] formal definition of agent interdependence and Johnson et al.'s [4] informal work on task interdependence.

Castlefranchi at al. [1] formally define non-social and social dependence, and describe the complex patterns of dependence relationships, such as unilateral and bilateral dependence, AND-Dependence, and OR-Dependence. We go further than Castelfranchi et al.'s formal definitions of agent interdependence [1] by considering soft agent interdependence. In Castelfranchi et al.'s formalism, a dependence exists from agent $a_{1}$ to agent $a_{2}$ if and only if $a_{1}$ can only complete the task with $a_{2}$. By considering utility as the measure of task completion, we generalise agent interdependence, allowing us to capture Castelfranchi et al.'s notion of (inter)dependence as hard (inter)dependence (utility for $a_{1}$ is 0 ), as well as to consider a notion of soft (inter)dependence (utility for $a_{1}$ is non-zero, but less than the composed case). Castlefranchi et al. consider that bilateral dependence can either be mutual or reciprocal, differing based on whether the agents' goals are shared (mutual) or separate (reciprocal). We have also considered the treatment of goal interdependence, but our definitions and analysis are omitted from this paper for space reasons.

Johnson et al. [4] looked at numerous existing definitions of interdependence, including the work of sociologists [10], and we build on their final definition of task interdependence. We believe that their definitions of interdependence are equivalent to our formal definitions, including their notion of soft and hard interdependence, although their definitions are informal, so these equivalences are not straightforward to assess. However, they do not explicitly consider agent or goal interdependence.

\section{Conclusion and future work}

Existing literature $[1,4]$ makes a strong case that interdependence is an important concept for the design of human agent collaboration. In this paper, we claim that it is useful to consider the different types of interdependence, namely agent and task interdependence, and we provide semi-formal definitions of these. The 
example briefly illustrates how knowledge of the type of interdependence can assist designers in choosing an appropriate agent coordination mechanism. This indicates that there is a link between interdependence and coordination mechanisms, and support our view of the benefit to having a fine-grained understanding of interdependence.

Our motivation for this work is to build autonomous agents that can collaborate with humans in a natural way. In other work, we are analysing other types of interdependence, such as goal and feedback interdependence [8], which are crucial for agents that learn. We also plan to investigate appropriate coordination mechanisms for each type of interdependence, and to explore the relationships between the different interdependence types, for example, the relationship between agent and task interdependence

\section{References}

[1] Castelfranchi, C., Miceli, M., Cesta, A.: Dependence relations among autonomous agents. In: Werner, E., Demazeau, Y. (eds.) Decentralized AI 3 - Proceedings of the Third European Workshop on Modelling Autonomous Agents and Multi-Agent Worlds (MAAMAW-91). pp. 215-227. No. 3 (1992)

[2] Hoare, C.: Communicating Sequential Processes. Prentice-Hall (1985)

[3] Johnson, M., Bradshaw, J., Feltovich, P., Jonker, C., Riemsdijk, B.V.: Autonomy and Interdependence in Human-Agent-Robot Teams. IEEE Intelligent Systems 27(2), 43-51 (2012)

[4] Johnson, M., Bradshaw, J., Feltovich, P., Jonker, C., Riemsdijk, M.B.V.: Coactive Design : Designing Support for Interdependence in Joint Activity. Journal of Human-Robot Interaction 3(1), 43-69 (2014)

[5] Parunak, H.V.D., Brueckner, S., Fleischer, M., Odell, J.: Co-x: Defining what agents do together. In: Proceedings of the AAMAS02, workshop on teamwork and coalition formation. pp. 62-69 (2002)

[6] Pfau, J., Miller, T., Sonenberg, L., Kashima, Y.: Logics of Common Ground (2014), http://people.eng.unimelb.edu.au/tmiller/ pubs/logics-of-common-ground.pdf, (Under review)

[7] Puranam, P., Raveendran, M., Knudsen, T.: Organization Design: The Epistemic Interdependence Perspective. Acad. of Management Review 37(3), 419-440 (2012)

[8] Saavedra, R., Earley, P.C., Van Dyne, L.: Complex interdependence in taskperforming groups. Journal of Applied Psychology 78(1), 61-72 (1993)

[9] Sichman, J.S.: DEPINT: Dependence-Based Coalition Formation in an Open Multi-Agent Scenario. JASSS 1(2) (1998)

[10] Thibaut, J.W., Kelley, H.H.: The social psychology of groups. John Wiley, Oxford, England (1959)

[11] Thompson, J.D.: Organizations in action. McGraw Hill, New York (1967)

[12] Van De Ven, A.H., Delbecq, A.L., Koenig, R.: Determinants of Coordination Modes within Organizations. American Soc. Review 41(2), 322-338 (1976)

[13] Winskel, G., Nielsen, M.: Models for concurrency. DAIMI Report Series 22(463) (1993) 